\title{
A greedy randomized adaptive search procedure application to solve the travelling salesman problem
}

\author{
Alvaro Neuenfeldt Júnior \\ Federal University of Santa Maria, Av. Roraima 1000, building 7, room 302, 97105900, Santa Maria, Brazil \\ alvaroj.eng@gmail.com \\ Lucas Rebouças Guimarães \\ Federal Institute of Education, Science and Technology of Espírito Santo, Av. Rio Branco 50, 29056-255, Vitória, \\ Brazil \\ lucasreb@gmail.com
}

Received (05-MAR-2019); Revised (04-SEP-2019); Accepted (6-SEP-2019); Published online (9-SEP-2019)

\begin{abstract}
The main objective of this article is to show an algorithm capable to find a minimal total length evaluation function roundtrip in symmetric Travelling Salesman Problem (TSP). Application of concepts related to Greedy Randomized Adaptive Search Procedure (GRASP) metaheuristics, with a $2 D$ Euclidean distance between nodes and return to start point. It was implemented through two instances defined in the TSPLIB. As results, it was observed that in all iterations the value of the initial solution, such as constructive heuristic, minimized over the course of local search optimization process, developed by the assumptions of the 2-Opt heuristics, where the best results found (836909 $\mathrm{km}$ ) was established for simulation considering a total of 5000 iterations. The traditional TSP solution has a strong implication in determining product delivery routes for customers established in different locations, inspired by the vendors' need to deliver products in different locations (the cities) using a shorter route, reducing the time required for the trip and the possible costs. Furthermore, the use of efficient TSP resolution methods can be adapted to applications found in industrial process practices, such as determining the delivery routes of materials to traverse a range of sectors from a common distribution center.
\end{abstract}

Key words: Greedy Randomized Adaptive Search Procedure (GRASP), Metaheuristics, Operational research, Travelling Salesman Problem (TSP).

\section{INTRODUCTION}

During the last fifty years the Travelling Salesman Problem (TSP) is considered one of the most preeminent themes to be explored into the operational research optimization problems, mainly because of its purest formulation nature, focused in some of the most important applications related to the practical activities, such as, for example, on some industrial planning [1,2], cargo logistics [3,4], vehicle routing and machine setups areas.

Therefore, considering a list of $n$ nodes and an evaluation criterion (e.g. distance, costs) between the subsequently pair of each one, the focus of TSP is to describe the optimum value capable to represent the best route option which can be covered without repetitions [5]. One of the most studied cases of TSP is related to solve problems that considering a 2D Euclidean distance between the nodes, mainly because of the relation with real situations into a transportation and logistics context, but also, because TSP is considered as a NP-hard computational problem not easy to solve in practical applications [6-8, 26].

Even because of its computational implementation characteristics, actually exists a large number of heuristics and exact methods that corresponds to solve this problem, such as Branch and bound, Branch and cut $[4,9]$, Dynamic programming $[2,10]$, Mixed integer programming [1,11], Genetic algorithms [12-15], Multiobjective evolutionary algorithms [16], Simulated annealing [17], Tabu search [18-19], Meta-heuristic for randomized priority search [3] and Greedy Randomized Adaptive Search Procedure (GRASP) [20,21,27], mostly based in the main principle that is reduce the necessary length to travel through all nodes (sometimes considered as cities) and return to initial checkpoint [5]. Considered an iterative process, the GRASP algorithm can be developed in two steps: the construction of greedy procedures to generate feasible solutions to the problem, by ordering elements based on the best maximization solution value obtained; and a local search improvement heuristic procedure, trying to 
improve the quality of the solutions at each iteration in a randomize sequence [21,22]. The best solution not necessarily will be the next to be changed, escaping the local optima.

Based on the assumptions described, the main objective of this present research is to develop an algorithm capable to find a minimal total length evaluation function roundtrip in symmetric TSP problems, through the application of the concepts related to GRASP metaheuristics, with a 2D Euclidean distance between the nodes and return to the original start point.

The traditional TSP solution has a strong implication in determining product delivery routes for customers established in different locations, inspired by the vendors' need to deliver products in different locations (the cities) using a shorter route, reducing the time required for the trip and the possible costs. Furthermore, the use of efficient TSP resolution methods can be adapted to applications found in industrial process practices, such as determining the delivery routes of materials to traverse a range of sectors from a common distribution center.

The research structure was designed in five topics that start mainly from the conceptual verification of some concepts and directives about TSP into a recent scientific research published. After, there are the definitions of the parameters used during the algorithm developed and specification of the TSPLIB instances used to support its implementation, which occurs in the last section of the present work.

\section{OVERVIEW OF TSP APPLICATIONS}

This section presents an overview of five researches developed during the last years about TSP, in a manner to bring some different ways in which the topic can be approached.

According to [23], a heuristic method very studied in literature related to TSP is the nearest neighbour algorithm. It consists in a simple and straightforward way to make a Hamiltonian cycle, starting by a city and taking step by step, the next closest city until the last city, when the cycle is closed by linking the last city to the first.

Firstly, a Lin-Kernighan heuristic to solve TSP problems was implemented by implemented [5], in sense to use a larger and more complex search steps than a previous original described in [24]. For instances, it was used a total of 7397 cities from TSPLIB, considered the largest nontrivial problem instance solved to optimality in 2000 , but the algorithm was capable to reproduce a scenario that involves, until, 85900 cities. At the end, it was observed that the computational experiments that have shown by this algorithm were highly effective, with an optimal solution obtained for all problems developed. Also, the running times were satisfactory for all tests problem and seem to be feasible for problems with fewer than 100000 cities.

For [7], the interest was using empirical methods to characterize the empirical complexity of the 2D Euclidean TSP, mainly because of its status to be a particularly prominent and well-studied NP-hard computational problem. For this, a total of 29 instances from Random Uniform Euclidean (RUE) and TSPLIB were applied to Concorde branch and cut solver heuristics. As findings, they demonstrate that some of the most widely studied types of TSP instances tend to be much easier than expected from previous theoretical and empirical results. The result suggests that the behaviour observed for the simple random structure underlying RUE is very similar to that obtained on the structured instances arising in various applications.

A six 2-Opt GRASP heuristics was proposed by [21] for several path-relinking strategies and solve the Symmetric Euclidean Clustered Traveling Salesman Problem (SECTSP), to find a minimum Hamiltonian cost cycle that the vertices of each cluster. However, there were no instances available to solve the SECTSP, in order of the necessity of develop alternatives versions for this purpose, that forces the generation of a set of six generic types. At the end, the proposed heuristics "G" and "GPR1PR2" were also compared to a Genetic Algorithm developed for solving the SECTSP without specifying the order of visiting the clusters.

A case study to investigates the development and application of a general Metaheuristic for Randomized Priority Search (Meta-RaPS) to TSP was developed by [3], focused in a large truck route assignment model developed by a trucking company, that estimates a more than $50 \%$ reduction in engineering time and over $\$ 2.5$ million annual savings in transportation costs using compared to their current method.

Finally, a new modified version of GRASP, called Multiple Phase Neighborhood Search-GRASP (MPNSGRASP) was proposed by [20], in order to improve its performance and to face the major disadvantage of normal GRASP which does not have a stopping criterion against the iterations that give minor improvements in the result. It was used for simulation 74 Euclidean sample problems with sizes ranging from 51 to 85900 nodes from TSPLIB.

\section{PARAMETERS AND INSTANCES FOR TSP}

The TSP was composed by four parameters that are critical to its characterization during the construction of the algorithm: The definition of the distances $\left(d_{c c r}\right)$ symmetry of each nodules $c$ (initial) and $c^{\prime}$ (final) path considered at the simulation $\left(d_{c c r}=d_{c r c}\right)$; the activation of each node only once per iteration; the starting and ending points are considered the same; and all cities are connected to each other, as to enable the filling of $n$ possible tour, as shown in Figure 1.

The optimization process of the $n$ following possibilities may be obtained through the simulation of the TSP can be described according to the objective function described in (1), from the function of verification able to minimize $D_{s}$ to a minimum value, that satisfies the restrictions imposed on the problem, which results in sequencing paths considered ideal. 


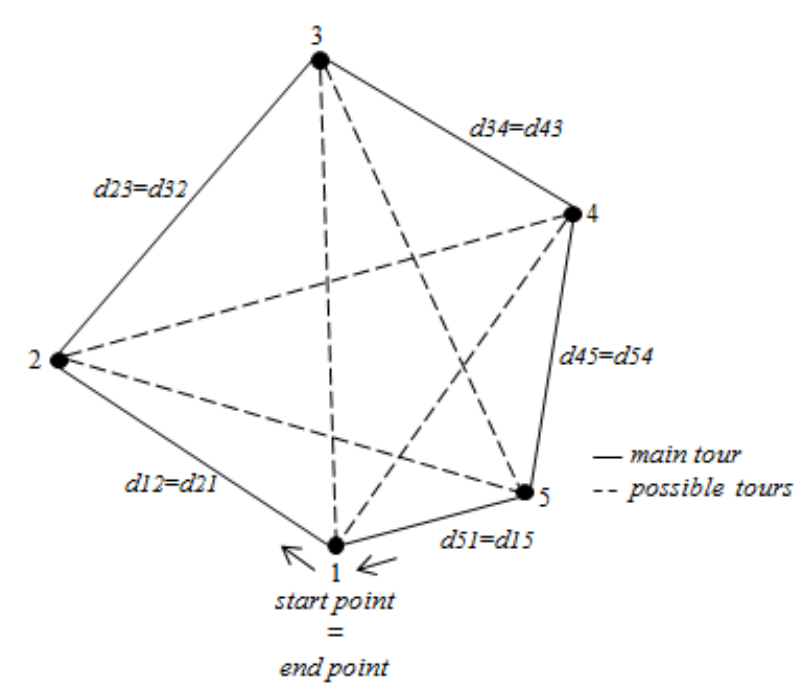

Figure 1. Example of the TSP structure adopted.

Minimize $D_{S}$

$$
\text { s.t.: }
$$

$D_{s}=\sum_{c=1}^{m} d_{c c r} \forall c \neq c^{\prime}$

$$
\begin{aligned}
& d_{c c t}=\sqrt{\left(c_{x}-c_{x}^{\prime}\right)^{2}+\left(c_{y}-c^{\prime}\right)^{2}} ; \\
& c_{x}, c_{x}^{\prime}, c_{y}, c_{y}^{\prime} \geq 0 ; \\
& c^{\prime}=\left(c_{x}^{\prime}, c_{y}^{\prime}\right) .
\end{aligned}
$$

For:

$c$ : starting point of the measured path, $\forall c \in\{0, \ldots, m-1\}$;

$c^{\prime}$ : end point of the measured path, $\forall c^{\prime} \in\{1, \ldots, m\}$;

$c_{x}: x$-coordinate of the starting point location in the cartesian plane;

$c_{x}^{\prime}: x$-coordinate of the end point location in the cartesian plane;

$c_{y}$ : $y$-coordinate of the starting point location in the cartesian plane;

$c^{\prime} y$ : $y$-coordinate of the end point location in the cartesian plane;

$d_{c c r}$ : Euclidean distance between the initial and final points evaluated;

$s$ : solution index.

For analysis and verification of behavior and level at which the results of the developed algorithm are established, it was proposed the complete utilization of one instance for benchmark developed by TSPLIB for Uruguay TSP simulation, composed of 734 cities [25].

\section{GRASP APPROACH}

As explained above in the introductory section, the develop of GRASP basically involves the development of two data parameterization steps, the constructive heuristics and local search, each responsible for the process optimization $D_{s}$ evaluation function for considered problem.
The constructive iterations process was initially proposed by random selection of the first city $(c=0)$ to be subjected to evaluation, in order to be checked in which case the value of its Euclidean distance to other cities is the smallest available, forming the first pair of shifts. This process occurs for the remaining cities to reach the last part of the tour, which relates to the initial point $m(c=0)$, where it is feasible to calculate the minimization $\left(D_{1}\right)$ for this first iteration.

For $N c$ a finite set of nodes in a manner that $D_{s}(c)$ is the current TSP solution sequence for the module value $D_{s}$ and an initial random first solution $\left(D_{1}\right)$ in relation to the objective of minimize the value of objective function. The 2-Opt local search heuristics (Figure 2) helps to seeks to change this initial result, in order to promote a comparison of these in relation to $D_{n}(s \leq n)$ other solutions, due the synchronized movement between two pairs of neighbor nodes.

However, are only considered those exchanges that are equal or reduce the optimization module measure adopted, such that $D_{s}$ is considered optimal when there is no possibility of exchanging able to satisfy its condition $[21,24]$.
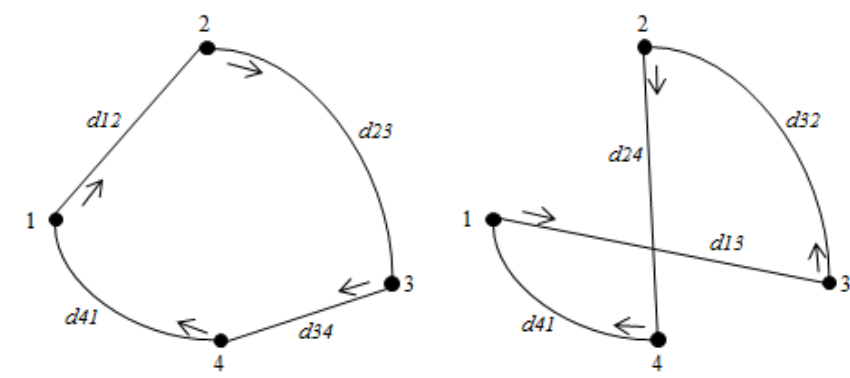

Figure 2. 2-Opt heuristics scheme. Source: [24].

Based on the procedures presented above, with the first $D_{1}$ solution already established, the second step of the algorithm is focused on the development of new solutions by double exchange between the cities of the first sequencing, through a finite count $(g)$ iterations, to generate $n$ solutions for the case. As a gradual process to search the optimal distance for the tour, every new solution found is compared with the previous ideal solution, so that, if the new solution is smaller, then take the originally assigned to former position and is considered as a new ideal solution.

In contrast, if the new value is higher, there is still the possibility of this being selected as the solution of the problem, through the randomize number (from 0 to 1 ) $\left(r_{s}\right)$ and the probability $(p)$ previously defined before the simulation (at the same manner from zero to 1 ). If $r_{s} \geq p$, so the new value should be stored in place of the previous one. Otherwise, the status remains unchanged until a new iteration is fostered.

After completion of the local search described above, there is a consolidation of data minimization function $D_{s}$, in a manner that can be possible the realization that what solution ongoing of iterations satisfied the problem and the route which was regarded as TSP ideal for the instances used. 


\section{RESULTS}

Through methodological procedures elucidated above and simulation parameters set out in section 3 , there was the possibility of performing the simulation of GRASP for the TSP instance that refers to all 734 cities of Uruguay, such a way that the results are presented as shown in the proposed chart in Figure 3 , for $g=1$ to $g=5000$ iterations. This presented approach was developed in the Integrated Development Environment IDE Microsoft Visual Studio, using the $\mathrm{C}_{++}$language. The results were generated on a computer with the following configuration: Processor Intel Core i7-4710 HQ $3.5 \mathrm{GHz}, 8 \mathrm{~GB}$ RAM Memory.

Two distance measurements were adopted as reference for the case the first is related to viable solution found in the heuristics constructive step, while the second reflects the shortest distance to the course of $n$ simulated iterations in the local search and considered as the lowest value found for TSP after each simulation.

According to the expectations established previously, the first observation can be given is that minimization of the initial solution obtained through constructive heuristics are not directly related to the number of iterations, as can be seen between $g=500$ and $g=2000$ in the graph. However, the relation holds true if related to the distance minimization of the end of full GRASP implementation (after the local search).

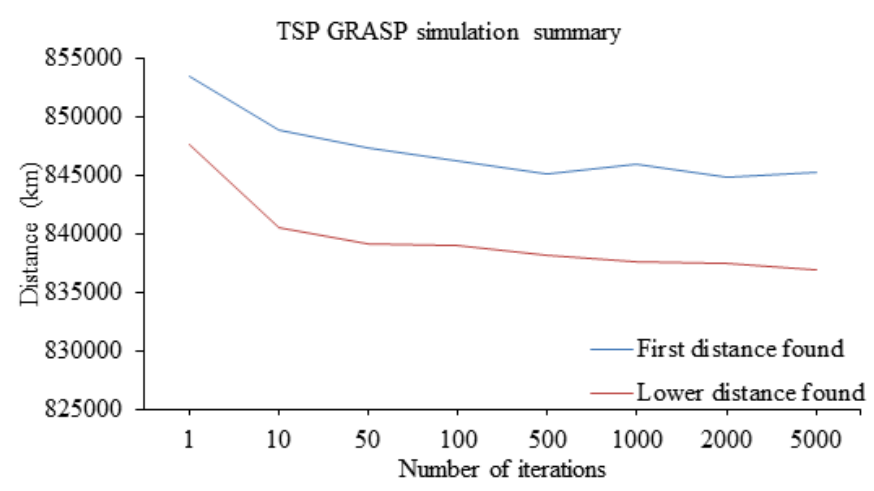

Figure 3. Simulations results for Uruguay TSP instance.

Next, it was necessary to check the behavior of the time consumed for processing each iteration proposed, the results of which are shown in the graphical representation in Figure 4.

As expected by checking the data, it could be observed the consolidation of the time growth in direct relationship with the total iterations adopted for the case. While in $g=1$ the time required is less than $1 \mathrm{~s}$, for $g=100$ this rises to $17.8 \mathrm{~s}$, an increasing trend time consumed in the first position.

With the number of iterations adopted, it was not possible to visualize at what time the model can achieve a stabilization of the results, but is gauged that it should be close to the $D_{8}=836909 \mathrm{~km}$ mark, found for the simulation developed with $g=5000$ on, approximately, $900 \mathrm{~s}$ of processing. Also, it is believed that regardless of the number of iterations attributed to $D_{s}$ mark shall not exceed a value of less than 835000 $\mathrm{km}$, given the profile of results and parameters used for the design of the algorithm proposed.

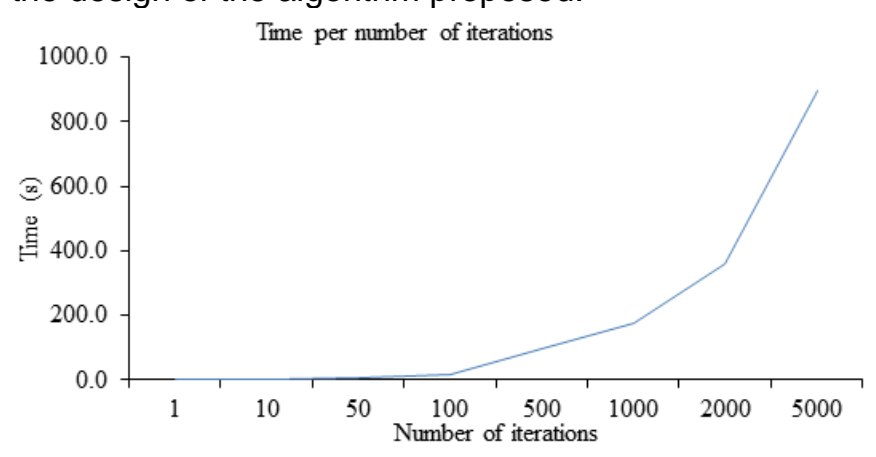

Figure 4. Time consumed for each number of iterations.

\section{CONCLUSION}

According to the main research objective that seeks the develop an algorithm capable to optimize the minimal total length evaluation function roundtrip in symmetric TSP problems, through the application of the concepts related to GRASP metaheuristics, it is possible affirm that the results demonstrated the capacity of mathematical programming developed to fulfill its purpose. Thus, it was observed that in all iterations the value of the initial solution was minimized over the course of local search optimization process, developed by the assumptions of the 2-Opt heuristics, where the best results found $\left(D_{8}=836909 \mathrm{~km}\right)$ is established for simulation considering a total of $g=5000$ iterations.

As limitations of research, it can be considered the use of symmetry between the one-way distances to and from each trip between cities, as well as the use of only one instance for simulation, which makes it infeasible to verify the robustness level of the heuristic adopted. Another point was the long time required for the GRASP fulfill its TSP implementation routines, which makes recommendable the use of a processing number not very extensive, but that the same time allow to obtain satisfactory results unless to conditions of the problem.

For future studies, it is expected that the proposed TSP presented is subjected to other methods of heuristics and metaheuristics, in order to seek more efficient solutions to instances subject to verification.

\section{REFERENCES}

[1] Cao, D.; Chen, M. (2005). A mixed integer programming model for a two line CONWIP-based production and assembly system. International Journal of Production Economics. 95(3), 317 - 326.

[2] Gromicho, J.A.S.; Hoorna, J.J.; Gamac, F.S.; Timmer, G.T. (2012). Solving the job-shop scheduling problem optimally by dynamic programming. Computers \& Operations Research. 39(12), $2968-2977$.

[3] DePuy, G.W.; Moraga, R.J.; Whitehouse, G.E. (2005). MetaRaPS: a simple and effective approach for solving the traveling salesman problem. Transportation research. Part E, Logistics and transportation review. 41(2), $115-130$.

[4] Côté, J.F.; Archetti, C.; Speranza, M.G.; Gendreau, M.; Potvin, J.Y. (2012). A branch-and-cut algorithm for the pickup and delivery traveling salesman problem with multiple stacks. Networks. 60(4), $212-226$. 
[5] Helsgaun, K. (2000). An Effective Implementation of the LinKernighan Traveling Salesman Heuristic. European Journal of Operational Research, 126(1), 106-130.

[6] Pizzolato, N.D.; Canen, A.G. (1998). Case study: improving industrial competitiveness: a TSP approach. Logistics Information Management, 11(3), 188-191.

[7] Hoos, H.; Stützleb, T. (2014). On the empirical scaling of runtime for finding optimal solutions to the travelling salesman problem. European Journal of Operational Research. 238(1), 87 $-94$.

[8] Santa Chávez, J. J., Echeverri, M. G., Escobar, J. W., \& Meneses, C. A. P. (2015). A Metaheuristic ACO to Solve the Multi-Depot Vehicle Routing Problem with Backhauls International Journal of Industrial Engineering and Management (IJIEM), 6(2), 49-58.

[9] Borne, S.; Mahjoub, A.R.; Taktak, R. (2013). A branch-and-cut algorithm for the Multiple Steiner TSP with Order constraints. Electronic Notes in Discrete Mathematics. 41 (5), 487 - 494.

[10] Malandraki, C.; Dial, R.B. (1996). A restricted dynamic programming heuristic algorithm for the time dependent traveling salesman problem. European Journal of Operational Research, $90(1), 45-55$.

[11] Alle, A.; Pinto, J.M. (2002). Mixed-Integer Programming Models for the Scheduling and Operational Optimization of Multiproduct Continuous Plants. Ind. Eng. Chem. Res., 41(11), 2689 - 2704.

[12] Chatterje, S.; Carrera, C.; Lynch, L.A. (1996). Genetic algorithms and traveling salesman problems. European Journal of Operational Research. 93(3), $490-510$.

[13] Schmitt, L.; Amini, M. (1998). Performance characteristics of alternative genetic algorithmic approaches to the Traveling Salesman Problem using path representation: An empirical study. European Journal of Operational Research, 108(3), 551580.

[14] Carter, A.E.; Ragsdale, C.T. (2006). A new approach to solving the multiple traveling salesperson problem using genetic algorithms. European Journal of Operational Research. 175(1), $246-257$.

[15] Zakir, A. (2014). The Ordered Clustered Travelling Salesman Problem: A Hybrid Genetic Algorithm. The Scientific World Journal, 1 - 13

[16] Wang, P.; Sanina, C.; Szczerbickib, E. (2015). Evolutionary algorithm and decisional DNA for multiple Travelling Salesman Problem. Neurocomputing, 150, $50-57$.

[17] Yip, P.P.C.; Pao, Y.H. (1995). Combinatorial optimization with use of guided evolutionary simulated annealing. IEEE Transactions on Neural Networks, 6(2), $290-295$.

[18] Gendreau, M.; Laporte, G.; Semet, F. (1998). A Tabu Search heuristic for the undirected selective traveling salesman problem. European Journal of Operational Research. 106(2-3), 539 545.

[19] Voudouris, C.; Tsang, E. (1999). Guided local search and its application to the traveling salesman problem. European Journal of Operations Research, 113 (1), $469-499$.

[20] Marinakis, Y.; Migdalas, A.; Pardalos, P.M. (2005). Expanding Neighborhood GRASP for the Traveling Salesman Problem. Computational Optimization and Applications, 32 (3), 231-257.

[21] Mestria, M.; Ochi, L.S.; Martins, S. L. (2013). GRASP with path relinking for the symmetric Euclidean clustered traveling salesman problem. Computers \& Operations Research. 40(12), $3218-3229$.

[22] Ribeiro, C.C.; Hansen, P. (2002). Essays and Surveys on Metaheuristics. Norwell: Kluwer Academic Publishers.

[23] Karkory, F. A.; Abudalmola, A. A. (2013). Implementation of Heuristics for Solving Travelling Salesman Problem Using Nearest Neighbour and Minimum Spanning Tree Algorithms. International Journal of Mathematical, Computational, Physical and Quantum Engineering 7(10), 987 - 997.

[24] Lin, S.; Kernighan, B.W. (1973). An Effective Heuristic Algorithm for the Traveling-Salesman Problem. Oper. Res. 21, 498 - 516.

[25] TSPLIB. Benchmark: TSPLIB instances - Uruguay National TSPs. Acessed 09 January 2019. http://www.math.uwaterloo.ca/tsp/world/uy734.tsp.

[26] Oliveira, J. F.; Neuenfeldt Júnior, A.; Silva, E.; Carravilla, M. A. (2016). A survey on heuristics for the two-dimensional rectangular strip packing problem. Pesquisa Operacional 36(2), $197-226$.

[27] Júnior, A. N.; Silva, E.; Gomes, A. M.; Soares, C.; Oliveira, J. F. (2019). Data mining based framework to assess solution quality for the rectangular 2D strip-packing problem. Expert Systems with Applications, 118, $365-380$. 